\title{
Serious events following COVID-19 vaccination with ChAdOx1 nCoV-19 vaccine (Vaxzevria): a short case series from Iran
}

\author{
Nasim Khajavirad ${ }^{1}$, Mohammadreza Salehi ${ }^{1}$, Abdolkarim Haji ghadery ${ }^{1}$, Hossein Khalili ${ }^{2}$, \\ Mehran Arab Ahmadi ${ }^{1}$, Seyed Ali Dehghan Manshadi ${ }^{1}$, and Ali Zare Dehnavi ${ }^{1}$ \\ ${ }^{1}$ Tehran University of Medical Sciences \\ ${ }^{2}$ Tehran University of Medical Sciences School of Pharmacy
}

December 2, 2021

\begin{abstract}
The COVID-19 vaccination programs have sparked several concerns and ongoing debates over safety issues. Here, we presented three cases of patients with serious adverse events, encephalopathy; Vaccine induced thrombotic thrombocytopenia, and Leukocytoclastic Vasculitis, after receiving the ChAdOx1 nCoV-19 vaccine.
\end{abstract}

\section{Introduction}

The coronavirus disease (COVID-19) contributed to a worldwide crisis causing major morbidity and mortality as well as devastating economic and social consequences. As for previous infectious diseases, vaccination is considered vital to control the COVID-19 pandemic. With unprecedented speed, several vaccines were developed and licensed internationally. By July 2021, the U.S. Food and Drug Administration (FDA) approved two mRNA-based vaccines: mRNA1273 (Moderna) and BNT162b2 (BioNTech/Pfizer); and one recombinant vector-based vaccine: adenovirus type 26 vector Covid-19 Vaccine Janssen (Johnson\&Johnson). In addition to previous vaccines, the European Medicines Agency (EMA) also approved the recombinant chimpanzee adenoviral vector vaccine ChAdOx1 nCoV-19 (Vaxzevria / previously COVID-19 vaccine AstraZeneca).

Several surveys have discovered various systematic, focal, and organ-specific adverse effects resulting from different vaccines(1)(2). Among several side effects of vaccines, concerns about unusual thrombotic events following COVID-19 vaccination with AstraZeneca started to grow early in March 2021. Several researchers from Norway(3), Germany(4), and United Kingdom(5) reported a group of patients who had been admitted within three weeks of AstraZeneca vaccine inoculation with thrombocytopenia and cerebral venous sinus thrombosis (CVST). In terms of neurological adverse effects, headache, decrease in mental attention, paralysis or weakness of extremities, dizziness, paraesthesia, and numbness were the most prevalent ones(1)(6). Complaints such as loss of consciousness, seizures, and facial weakness were rare, though(1). Skin manifestation of COVID-19 vaccination ranged wide spectrum including chilblain-like, urticarial, vesicular, maculopapular, livedoid and vasculitic lesions(7)(8). Thus, it seems that COVID-19 vaccines' have a wide spectrum of adverse effects and further investigations are needed to help clinicians for better diagnosis and management.

The current paper aims to report three post-AstraZeneca COVID-19 vaccination events causing hospitalization, including encephalopathy, CVST, and Leukocytoclastic Vasculitis (LCV).

\section{Patients and methods}

\section{Case1. Post vaccination encephalopathy}

The patient was a 56-year-old female laboratory technician with no remarkable medical history and drug consumption. She was admitted to the hospital with complaints of confusion, decreased level of consciousness, 
and some degree of disorientation. She had received the first shot of the AstraZeneca COVID-19 vaccine one week before the admission. Two days after vaccination, her symptoms initiated with general weakness, myalgia, headache, and low grade fever that gradually worsened. The symptoms continued with decrease in consciousness, disorientation, bizarre behaviors and agitation during 72 hours that, eventually led to hospital admission. At the time of hospital admission, the patient was confused, and her neurological examination demonstrated disorientation to time, place, and person. She also had impairment in gait and the finger-tonose test. Glasgow coma scale revealed E3M5V3; (an eye (3), verbal (3), and motor (5) response). Other neurological examinations (i.e., sensory, cranial nerves, and speech) were normal. Her vital signs were stable except for fever $\left(38.6{ }^{\circ} \mathrm{C}\right)$.

With clinical suspicious of viral encephalitis, lumbar puncture was done and intravenous acyclovir, $10 \mathrm{mg} / \mathrm{kg}$ every 8 hours was started. The patient's orientation and consciousness improved within less than two days. Cerebral spinal fluid (CSF) was clear and colorless with a total WBC count of $<5$ cells $/ \mathrm{mm}^{3}$, a normal glucose level, and significantly raised protein concentration of $119 \mathrm{mg} / \mathrm{dl}$. However, CSF viral panels (HSV1, HSV2, CMV, EBV, VZV, HHV6, HHV7, and HHV8 polymerase chain reaction (PCR) were negative. Acyclovir was discontinued. Further laboratory studies including complete blood count (CBC), blood electrolytes (Na, K, $\mathrm{P}, \mathrm{Ca}$, and $\mathrm{Mg}$ ), liver enzymes tests, urine analysis and culture, blood culture, blood and urine toxicology, blood gases test, coagulation tests, and SARS-COV-2 PCR were all unremarkable. She also underwent brain MRI (figure1) and abdominopelvic sonography that were normal. Rapidly, the patient's general condition was ameliorated and she was discharged two days later and referred to the outpatient clinic for a follow-up visit. Clinical and neurological conditions were normal at the follow-up visit one month later.

\section{Case2. Vaccine induced thrombotic thrombocytopenia (VITT)}

A 70-year-old female with a past medical history of diabetes mellitus type 2, hypertension, and coronary artery disease (had undergone percutaneous coronary intervention 10 years ago) received her first shot of COVID19 AstraZeneca vaccine in late May 2021(day 0). The following day she developed a generalized persistent headache that, despite consumption of acetaminophen, didn't improve. The next day she experienced a single episode of generalized tonic-clonic seizure that led to refer to the local hospital. During the hospital stay, laboratory findings revealed a mild leukocytosis (WBC: 12000/ $\mu \mathrm{L}$ ), mildly elevated Aspartate aminotransferase test (AST: 60U/L), an increased Lactic Acid Dehydrogenase (LDH: 630U/L) and a high creatine phosphokinase level (CPK: $450 \mathrm{mcg} / \mathrm{L}$ ) however the rest (BUN, Creatinine, ESR, CRP and urine analysis) were normal. The neuroimaging findings including brain computed tomography $(\mathrm{CT})$, magnetic resonance imaging (MRI) imaging, and magnetic resonance venography (MRV), were unremarkable. The patient was discharged five days later due to normal workups, no new seizure and amelioration of her headache.

After a few days, headache and convulsions commenced again, and their severity and frequency worsened gradually that finally led to hospitalization at our center after approximately two weeks (day 21). At the admission, the patient was lethargic and was experiencing seizure episodes 2-3 times per day, each one lasting 2-3 minutes. Clinical and neurologic examinations showed no remarkable findings, and all her vital signs were within a normal range (BP: 120/80, T: $36.8^{\circ} \mathrm{C}, \mathrm{HR}: 75$, RR: 14, O2sat:96\%). The primary ECG showed no pathologic findings. At this time, moderate thrombocytopenia $[78 \times 103 / \mathrm{ml}$; normal reference range $(\mathrm{NRR}) 150-450 \times 103 / \mathrm{ml}]$ with normal peripheral blood smear morphology, markedly elevated D-dimer (11 $\mu \mathrm{g} / \mathrm{ml}(<0.5)$ ), a fibrinogen level at the lower limit of the normal range, anemia $(\mathrm{Hgb}: 9.4 \mathrm{~g} / \mathrm{dl})$ and elevated inflammatory markers (ESR: $45 \mathrm{~mm} / 1 \mathrm{hr}$, CRP: $25 \mathrm{mg} / \mathrm{L}$ ) were detected. Other blood tests were normal. Thus, vaccine-induced thrombotic thrombocytopenia (VITT) was suggested(9) and, further investigations were requested.

The anti-PF4 IgG antibody ELISA tests was positive (380 ng/ml (42.1-313.40)). Brain CT venography findings were in favor of cerebral venous sinus (sagittal sinus) thrombosis (CVST), so the patient underwent brain MRI. In brain MRI, periventricular abnormal signals without diffusion restriction were seen in favor of small vessel, ischemic changes (FAZEKAS III) and T2 and diffusion-weighted signal changes representative of acute infarction was also visible in left occipital lobe. In brain MRV filling defect in favor of left transverse sinus thrombosis was seen (figure2). Finally, brain and cervical MRA were also unremarkable and no 
pathologic finding was observed.

During hospital stay, Intravenous immunoglobulin (IVIG) (1 $\mathrm{gr} / \mathrm{kg} /$ day for 2 days), corticosteroids (0.5 mg/kg prednisolone), rivaroxaban (15 mg/BID), sodium valproate and levetiracetam were started. Convulsions ceased within two days, and platelet began going up on the 3rd day. The patient's general condition gradually improved, seizures were controlled, the patient's D-dimer decreased, and platelets and hemoglobin returned to normal and after 10 days, the patient was discharged from the hospital with continued anticoagulants and anticonvulsants drugs.

\section{Case3. Leukocytoclastic Vasculitis(LCV)}

A 77-year-old female with hypertension (HTN) presented to our emergency room with complaint of a fiveday extensive rash and edema which was commenced two days after receiving her first shot of COVID19 AstraZeneca vaccine. At the admission, she had extensive palpable purpura and non-pitting edema on both lower extremities, below the knees (figure3). Rests of her examinations were unremarkable and the vital signs were within the normal range. Thus, she hospitalized and underwent further investigations. Initial laboratory tests revealed a pancytopenia (WBC: 1300/ml (neutrophil: 60\%, lymphocyte: 37\%), Hgb: $7.7 \mathrm{gr} / \mathrm{dl}$, Platelet: $75000 / \mathrm{ml})$, elevated erythrocyte sedimentation rate $(71 \mathrm{~mm} / \mathrm{h}$; normal reference range (NRR) $0-30 \mathrm{~mm} / \mathrm{hr}$ ), high lactic acid dehydrogenase $(\mathrm{LDH})(584 \mathrm{U} / \mathrm{L}$; normal reference range (NRR) $140-280 \mathrm{U} / \mathrm{L})$, an elevated NT-PRO-BNP level $(3780 \mathrm{pg} / \mathrm{ml})$ and a significantly elevated D-dimer $(2.5 \mu \mathrm{g} / \mathrm{ml}$; normal reference range $(\mathrm{NRR})<0.5 \mu \mathrm{g} / \mathrm{ml}$ ), however, the rest(CRP, FBS, LFT, BUN, Cr, urine analysis, albumin, fibrinogen and coagulation tests ) were normal. The examination of peripheral blood smear (PBS), revealed Rouleaux formation and platelet aggregation (figure3). Polymerase chain reaction (PCR) test for COVID19 was negative. Patients' characteristics are summarized in table 1.

Due to patients' clinical manifestations and laboratory findings a possibility of vasculitis was suggested. Prednisolone $(0.5 \mathrm{mg} / \mathrm{kg} /$ day $)$ prescribed and skin biopsy and further tests were ordered. HIV Ag/Ab and viral hepatitis panels were negative. Immunological screening including: C3, C4, CH50, ANA, Antids DNA, ANCA-C and ANCA-P were normal. Microscopic examination of skin specimen revealed vasculopathic changes characterized by perivascular lymphocytic infiltrate with few nuclear debris. Permeating into vessel wall with endothelia thickening and extravasated RBC. Foci of microhemorrhage in superficial dermis also identified. Purpuric vasculopathic reaction pattern of lymphocytic type in histopathology was compatible with purpric lymphocytic vasculitis diagnosis. Finally, one week after treatment rash and symptoms resolved, blood cells count improved (WBC: 4150, HB: 10.2, PLT: 110,000) and the patient was discharged.

\section{Discussion}

Common adverse effects of COVID-19 vaccination including local reactions, fever, myalgia, arthralgia and headache can be well tolerated in outpatient setting, but some conditions need inpatient care and somehow can be problematic or even fatal. Here in, we reported three cases of serious events of post COVID-19 AstraZeneca vaccination that hospitalized and managed successfully, including encephalopathy, CVST and LCV.

Causality assessment of adverse drug reaction (ADR) with COVID-19 vaccination based on ADR scales including Naranjo Probability Scale(10) and the WHO-UMC causality system(11) showed all discussed cases were categorized as probable but not definite cases caused by COVID-19 vaccination. Severity of ADR by COVID-19 vaccination for presented cases were evaluated by J Seigel and PJ Schneider study(12) and also Karch and Lasanga study(13), post vaccination encephalitis and VITT were classified as severe cases requiring intensive medical care which are life threatening reactions, while LCV categorized with moderate severity requiring special treatment with an increase in hospitalization by at least one day.

Encephalitis/encephalopathy is defined as inflammation of brain tissue provoked by an infection or an autoimmune response(14). The occurrence of encephalitis, according to several publicly existing databases (Welcome to GOV.UK (www.gov.uk), European Medicines Agency | (europa.eu), Paul-Ehrlich-Institut Startseite (pei.de)), estimated to be 8 and 2 per 10 million injected shots following AstraZeneca and Pfizer- 
Biontech vaccines, respectively(15). Possible or confirmed diagnosis of encephalitis is determined by combination of clinical manifestations, laboratory tests, neuroimaging, and electroencephalographic studies(16). Our first case had the major criteria of encephalitis according to Venkatesan et al., presenting to medical attention with altered mental status lasting [?]24 h with no alternative cause stated, and one minor criteria of documented fever within the 72 hours prior or after presentation(16). The patient neuroimaging and CSF analysis weren't compatible with encephalitis and EEG wasn't performed for her, so the complete criteria for encephalitis cannot be fulfilled and encephalopathy after vaccination may be more likely. However, it doesn't seem to be a just fever-related delirium due to patient's age, past medical history and her clinical picture and a CNS related dysfunction is highly probable.

Previously reported post vaccination encephalopathy cases were all older than 50 years of age except for one 21 year old female, and were more common following ChAdOx1 nCov-19 administration(15)(17)(18). Fever and myalgia were commonly seen. Alike our patient, brain imaging was normal in all of these reports and no remarkable finding was seen(15)(17)(18). Two cases had abnormal findings in EEG, a 77years old male showed moderate diffuse slowing and another 77year old male presented generalized slow background in the theta range(17)(18). In terms of CSF analysis, in contrast with our patient, pleocytosis was a common finding being seen in 4 out of 5 cases and only one patient had normal WBC count with an elevated protean level $(15)(17)(18)$.

VITT is well established side effect of AZ and many studies reported the post AZ vaccination VITT. A review by Franchini et al.(19) On previous VITT cases due to AZ vaccine showed thrombotic complications occurred 5-25 days after first dose of $\mathrm{AZ}$ vaccination and in majority of cases site of thrombosis was cerebral veins, while splenic vein thrombosis and pulmonary embolism were in second and third place. Our patient showed initial symptoms on first day after vaccination and admitted to hospital but discharged due to improvement of patient's conditions. But on second admission, few days after discharge, CVST was detected on imaging and laboratory data confirmed the VITT by positive HIPA, PIPA, and anti-PF4 IgG ELISA tests.

Although vaccine-induced thrombotic thrombocytopenia (VITT) was first reported after AZ vaccination growing number of papers were recently published reporting the VITT with Johnson\&Johnson(20)(21), Moderna(21)(22), and BioNTech/Pfizer(21)(23) vaccines. Due to positive anti-PF4 antibodies in both mRNAbased vaccines and vector-based vaccines it raises the question which component of vaccine is responsible to elicit response to PF4 proteins and VITT phenomenon?

LCV is a cutaneous small vessel vasculitis of dermal capillaries and venules and typically presents with a painful, burning rash predominantly in lower extremities. It is often idiopathic but can be associated with infections, neoplasms, or medications(24). However, relation between vaccination or immunization with vasculitis is not well determined, vasculitis precipitation has been reported secondary multiple vaccines including influenza virus, hepatitis B virus (HBV), Basil Calmette-Guerin (BCG), and Human papillomavirus $(\mathrm{HPV})(25)$.

Skin vasculitis with cutaneous lesions have been reported during mild and fulminant COVID-19 disease(26). On the other hand, vasculitis was also reported after COVID-19 vaccination. A recent study by McMahon et al. on 417 cases of cutaneous reaction after mRNA COVID-19 vaccines, reported $0.7 \%$ and $2.9 \%$ of vasculitis after first dose of Moderna and BioNTech/Pfizer vaccines, respectively(27). Several case reports were also reported skin vasculitis following vector-based vaccines including AZ(28) and Johnson Johnson(29).

\section{Conclusions}

Due to different and diverse side effects of COVID-19 vaccine, it is important for healthcare providers and patients to be aware that COVID-19 vaccination could rarely precipitate serious side effects which caused hospitalization and extensive treatment.

\section{References:}

1. RAK K, R J, S P, SV M. Side effects of BNT162b2 mRNA COVID-19 vaccine: A randomized, crosssectional study with detailed self-reported symptoms from healthcare workers. Int J Infect Dis [Internet]. 
2021 May 1 [cited 2021 Aug 29];106:376-81. Available from: https://pubmed.ncbi.nlm.nih.gov/33866000/

2. Riad A, Pokorná A, Attia S, Klugarová J, Košč́k M, Klugar M. Prevalence of COVID-19 Vaccine Side Effects among Healthcare Workers in the Czech Republic. J Clin Med [Internet]. 2021 Apr 1 [cited 2021 Aug 29];10(7):1428. Available from: /pmc/articles/PMC8037149/

3. NH S, IH S, AE M, LA M, F L-J, MT A, et al. Thrombosis and Thrombocytopenia after ChAdOx1 nCoV-19 Vaccination. N Engl J Med [Internet]. 2021 Jun 3 [cited 2021 Aug 30];384(22):2124-30. Available from: https://pubmed.ncbi.nlm.nih.gov/33835768/

4. A G, T T, TE W, K W, PA K, S E. Thrombotic Thrombocytopenia after ChAdOx1 nCov-19 Vaccination. N Engl J Med [Internet]. 2021 Jun 3 [cited 2021 Aug 30];384(22):2092-101. Available from: https://pubmed.ncbi.nlm.nih.gov/33835769/

5. Scully M, Singh D, Lown R, Poles A, Solomon T, Levi M, et al. Pathologic Antibodies to Platelet Factor 4 after ChAdOx1 nCoV-19 Vaccination. https://doi.org/101056/NEJMoa2105385 [Internet]. 2021 Apr 16 [cited 2021 Aug 30];384(23):2202-11. Available from: https://www.nejm.org/doi/full/10.1056/NEJMoa2105385

6. Bae S, Lee YW, Lim SY, Lee J-H, Lim JS, Lee S, et al. Adverse Reactions Following the First Dose of ChAdOx1 nCoV-19 Vaccine and BNT162b2 Vaccine for Healthcare Workers in South Korea. J Korean Med Sci [Internet]. 2021 [cited 2021 Aug 30];36(17):1-9. Available from: /pmc/articles/PMC8093607/

7. C GC, A C, G CH, P R-J, D F-N, A R-VL, et al. Classification of the cutaneous manifestations of COVID19: a rapid prospective nationwide consensus study in Spain with 375 cases. Br J Dermatol [Internet]. 2020 Jul 1 [cited 2021 Aug 30];183(1):71-7. Available from: https://pubmed.ncbi.nlm.nih.gov/32348545/

8. AV M, G G, C M, V G, P V, V M, et al. The clinical spectrum of COVID-19-associated cutaneous manifestations: An Italian multicenter study of 200 adult patients. J Am Acad Dermatol [Internet]. 2021 May 1 [cited 2021 Aug 30];84(5):1356-63. Available from: https://pubmed.ncbi.nlm.nih.gov/33476725/

9. Aleem A, Nadeem AJ. Coronavirus (COVID-19) Vaccine-Induced Immune Thrombotic Thrombocytopenia (VITT). StatPearls [Internet]. 2021 Jul 18 [cited 2021 Aug 30]; Available from: https://www.ncbi.nlm.nih.gov/books/NBK570605/

10. CA N, U B, EM S, P S, I R, EA R, et al. A method for estimating the probability of adverse drug reactions. Clin Pharmacol Ther [Internet]. 1981 [cited 2021 Aug 30];30(2):239-45. Available from: https://pubmed.ncbi.nlm.nih.gov/7249508/

11. The use of the WHO-UMC system for standardised case causality assessment [Internet]. [cited 2021 Aug 30]. Available from: https://www.who.int/publications/m/item/WHO-causality-assessment

12. Preventability and severity assessment in reporting adverse drug reactions - PubMed [Internet]. [cited 2021 Aug 30]. Available from: https://pubmed.ncbi.nlm.nih.gov/1524068/

13. Adverse drug reactions. A critical review - PubMed [Internet]. [cited 2021 Aug 30]. Available from: https://pubmed.ncbi.nlm.nih.gov/1242749/

14. D D, SJ P, CR K, A M, AS L-C, VA L, et al. Autoimmune encephalitis epidemiology and a comparison to infectious encephalitis. Ann Neurol [Internet]. 2018 Jan 1 [cited 2021 Aug 30];83(1):166-77. Available from: https://pubmed.ncbi.nlm.nih.gov/29293273/

15. F Z, T G, R K, WR S, A R. Postvaccinal Encephalitis after ChAdOx1 nCov-19. Ann Neurol [Internet]. 2021 Aug 13 [cited 2021 Aug 30]; Available from: https://pubmed.ncbi.nlm.nih.gov/34324214/

16. A V, AR T, KC B, AS L, J S, A B, et al. Case definitions, diagnostic algorithms, and priorities in encephalitis: consensus statement of the international encephalitis consortium. Clin Infect Dis [Internet]. 2013 Oct 15 [cited 2021 Aug 30];57(8):1114-28. Available from: https://pubmed.ncbi.nlm.nih.gov/23861361/ 
17. L B, G A, A M, I P, S R, P C, et al. Hyperacute reversible encephalopathy related to cytokine storm following COVID-19 vaccine. J Neuroimmunol [Internet]. 2021 Sep 15 [cited 2021 Aug 30];358. Available from: https://pubmed.ncbi.nlm.nih.gov/34284342/

18. G T-A, JC M, Y H, CR G, MR S, SK A, et al. Acute encephalitis, myoclonus and Sweet syndrome after mRNA-1273 vaccine. BMJ Case Rep [Internet]. 2021 Jul [cited 2021 Aug 30];14(7):e243173. Available from: https://pubmed.ncbi.nlm.nih.gov/34312136/

19. Franchini M, Liumbruno GM, Pezzo M. COVID-19 vaccine-associated immune thrombosis and thrombocytopenia (VITT): Diagnostic and therapeutic recommendations for a new syndrome. Eur J Haematol [Internet]. 2021 Aug 1 [cited 2021 Aug 30];107(2):173-80. Available from: /pmc/articles/PMC8239516/

20. Muir K-L, Kallam A, Koepsell SA, Gundabolu K. Thrombotic Thrombocytopenia after Ad26.COV2.S Vaccination. https://doi.org/101056/NEJMc2105869 [Internet]. 2021 Apr 14 [cited 2021 Aug 30];384(20):1964-5. Available from: https://www.nejm.org/doi/full/10.1056/NEJMc2105869

21. Cines DB, Bussel JB. SARS-CoV-2 Vaccine-Induced Immune Thrombotic Thrombocytopenia. https://doi.org/101056/NEJMe2106315 [Internet]. 2021 Apr 16 [cited 2021 Aug 30];384(23):2254-6. Available from: https://www.nejm.org/doi/full/10.1056/nejme2106315

22. Sangli S, Virani A, Cheronis N, Vannatter B, Minich C, Noronha S, et al. Thrombosis With Thrombocytopenia After the Messenger RNA-1273 Vaccine. Ann Intern Med [Internet]. 2021 Jun 29 [cited 2021 Aug 30]; Available from: /pmc/articles/PMC8251935/

23. JS A-M, S AR, MS K, AM AH, H F, B AR, et al. A 59-Year-Old Woman with Extensive Deep Vein Thrombosis and Pulmonary Thromboembolism 7 Days Following a First Dose of the Pfizer-BioNTech BNT162b2 mRNA COVID-19 Vaccine. Am J Case Rep [Internet]. 2021 [cited 2021 Aug 30];22(1). Available from: https://pubmed.ncbi.nlm.nih.gov/34117206/

24. Leukocytoclastic Vasculitis - PubMed [Internet]. [cited 2021 Aug 30]. Available from: https://pubmed.ncbi.nlm.nih.gov/29489227/

25. C B, F T, P F, GS A, C S, NS B, et al. Vasculitis as an adverse event following immunization Systematic literature review. Vaccine [Internet]. 2016 Dec 12 [cited 2021 Aug 30];34(51):6641-51. Available from: https://pubmed.ncbi.nlm.nih.gov/26398442/

26. Casas CG, Catala A, Hernandez GC, Rodriguez-Jimenez P, Fernandez-Nieto D, Lario AR-V, et al. Classification of the cutaneous manifestations of COVID-19: a rapid prospective nationwide consensus study in Spain with 375 cases. Br J Dermatol [Internet]. 2020 Jul 1 [cited 2021 Aug 30];183(1):71-7. Available from: https://onlinelibrary.wiley.com/doi/full/10.1111/bjd.19163

27. DE M, E A, M R, JB L, D M, A T, et al. Cutaneous reactions reported after Moderna and Pfizer COVID-19 vaccination: A registry-based study of 414 cases. J Am Acad Dermatol [Internet]. 2021 Jul 1 [cited 2021 Aug 30];85(1):46-55. Available from: https://pubmed.ncbi.nlm.nih.gov/33838206/

28. L G-P, M P-P, D F-L, J M-M, C G-C, JL R-P, et al. Small-vessel vasculitis following Oxford-AstraZeneca vaccination against SARS-CoV-2. J Eur Acad Dermatol Venereol [Internet]. 2021 Aug 4 [cited 2021 Aug 30]; Available from: https://pubmed.ncbi.nlm.nih.gov/34310763/

29. Berry CT, Eliliwi M, Gallagher S, Panaccione S, Klein WM, Healy AL, et al. Cutaneous small vessel vasculitis following single-dose Janssen Ad26.COV2.S vaccination. JAAD Case Reports [Internet]. 2021 Sep [cited 2021 Aug 30];15:11. Available from: /pmc/articles/PMC8302840/

\section{Figure legends}

Fig.1 Patient1 brain MRI, (a \& b) normal T1 and T2 sequences

Fig.2 Patient2 brain MRI, (a) acute infarction in left occipital lobe, (b \& c) Filling defect in left transverse sinus representative of thrombosis is visible 
Fig.3 Patient3, (a \& b) Palpable purpura and non-pitting edema on lower extremities, (c) Platelet aggregation and Rouleaux formation in PBS
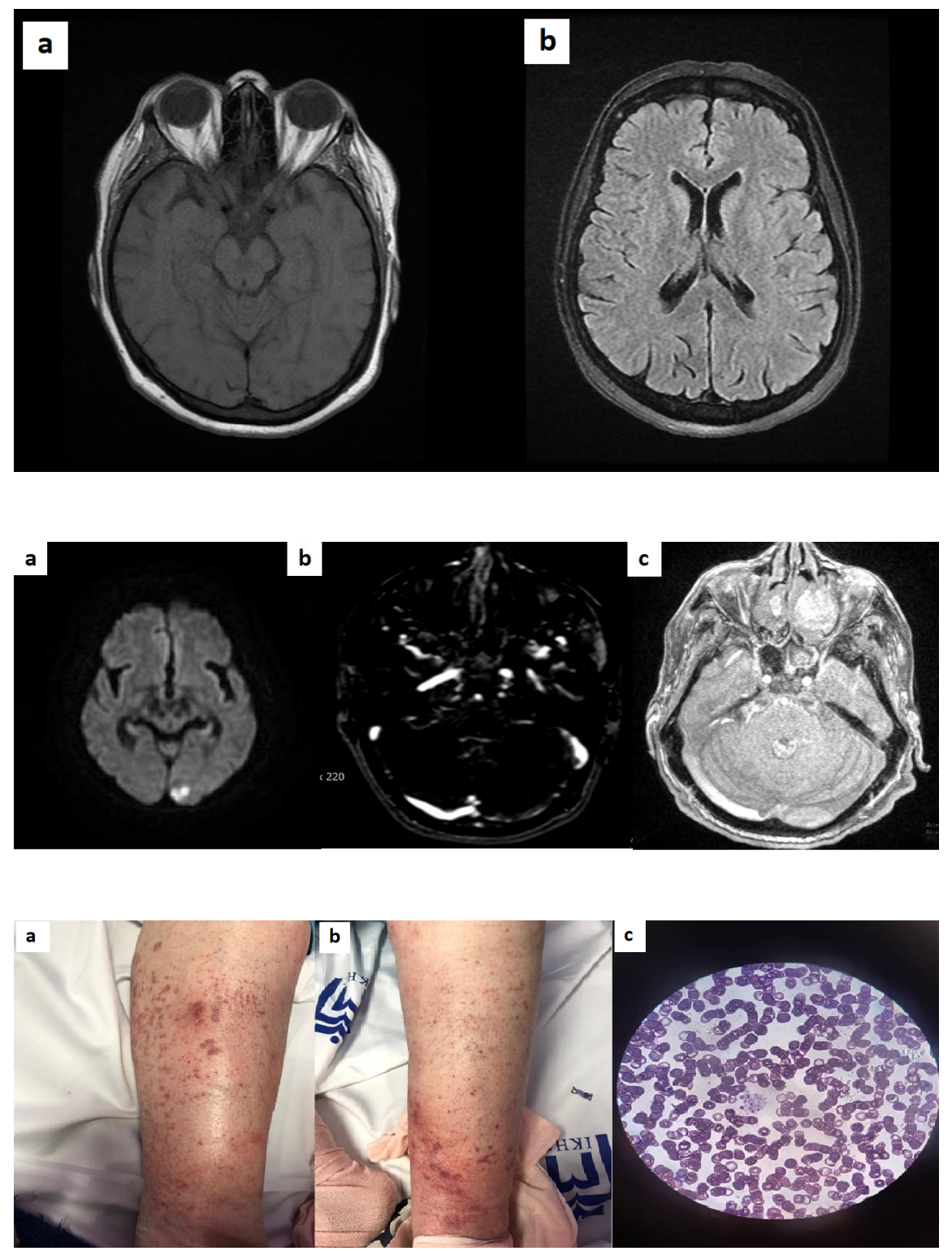